\title{
Efecto protector de una cepa no virulenta contra la infección con una cepa virulenta de Trypanosoma cruzi en un modelo murino"
}

\author{
Protective effect of a non virulent strain against infection of a highly virulent \\ strain of Trypanosoma cruzi in a murine model
}

\author{
C Zúñiga ${ }^{1 *}$, R Cepeda ${ }^{2}$, MT Paláu ${ }^{1}$, A Vera ${ }^{1}$, J Mejía $^{1}$, U Vergara ${ }^{1}$ \\ ${ }^{1}$ Departamento de Medicina Preventiva Animal. ${ }^{2}$ Departamento de Ciencias Biológicas, \\ Facultad de Ciencias Veterinarias, Universidad de Chile, Santiago.
}

\begin{abstract}
SUMMARY
This study was carried out to compare the infection course of Trypanosoma cruzi in the ACA inbred mouse strain inoculated with 2000 blood trypomastigotes of the Tulahuen strain and the Colombian Munantá strain. Parasitaemia and mortality rates showed different patterns for both strains of the parasite. All the Tulahuen infected mice died within 3 weeks after infection, in contrast, all mice infected with the Munantá strain survived, although they showed higher level of parasitaemia. These results are in agreement with previous findings suggesting poor correlation between death and parasitaemia level. The histopathological study showed different degrees of parasitism and inflammatory infiltrates in the heart, according to the parasite strain. The infection with the Munantá strain resulted in scarce parasitism and discrete inflammatory infiltrate. However, severe inflammation and tissue parasitism were observed in the Tulahuen infected mice. The protective effect of the non virulent Munantá strain against infection with the virulent Tulahuen strain was studied. When the Tulahuen strain was inoculated into mice after a challenge with the Munantá strain, parasitaemia was more controlled, and all the mice survived, showing lower inflammatory infiltration and a low number of pseudocysts. Application of these results to vaccine development is proposed.
\end{abstract}

Palabras clave: Trypanosoma cruzi, histopatología, estudio de protección.

Key words: Trypanosoma cruzi, histopathology, protection study.

\section{INTRODUCCION}

La enfermedad de Chagas o tripanosomiasis americana es causada por el parásito hemoflagelado Trypanosoma cruzi y afecta a millones de personas en Centro y Sudamérica (Dias y col 2002). Aunque la mayoría de los pacientes sobreviven a la etapa aguda de la enfermedad, en la etapa crónica desarrollan complicaciones secundarias severas, debido principalmente a cardiopatías y megasíndromes (Morris y col 1990, Botero y Restrepo 1992). T. cruzi es un parásito digénico, que presenta un ciclo de vida con alternancia entre hospederos invertebrados y vertebrados. En ambos casos presenta una baja especificidad, infectando a insectos de la familia Reduviidae y un amplio rango de mamíferos silvestres y domésticos, incluyendo al humano (Burleigh y Andrews 1995, Stevens y col 2001).

El modelo murino es uno de los modelos experimentales más utilizado en el estudio del desarrollo de la infección y la inducción de la respuesta inmune, frente a

Aceptado: 11.04 .2006

\# Financiado por Proyecto SIDA / SAREC

* Facultad de Ciencias Veterinarias y Pecuarias, Universidad de Chile. Correo 15 Casilla 2, La Granja, Santiago, Chile. clzuniga@uchile.cl este parásito, dado que diferentes cepas puras de ratones difieren en la susceptibilidad o resistencia a la infección (Postan y col 1983, Zúñiga y col 1998). Aunque se ha analizado el rol de diversos factores dependientes tanto del parásito (Urzúa y col 2004) como del hospedero (Hoft y col 1993, Zúñiga y col 2002), aún no se han identificado con certeza los factores directamente involucrados en los fenómenos de resistencia o susceptibilidad a la infección.

Para probar la hipótesis de que la infección con cepas no virulentas de T. cruzi altera el curso de la infección con cepas virulentas del parásito, en el presente trabajo se analizó el desarrollo de la infección en ratones de la cepa ACA infectados con 2.000 tripomastigotes sanguíneos de la cepa Tulahuen y en ratones infectados con la cepa Munantá de T. cruzi. Se realizó luego un estudio de protección, inoculando la cepa virulenta Tulahuen en ratones previamente infectados con la cepa no virulenta Munantá. En todos los ratones infectados se hizo un estudio de la evolución de la infección, determinando niveles de parasitemia, mortalidad acumulada y daño tisular.

\section{MATERIAL Y METODOS}

Parásitos. Las infecciones experimentales se realizaron utilizando tripomastigotes sanguíneos de $T$. cruzi de la 
cepa chilena Tulahuen aislada originalmente en la comuna del mismo nombre a partir de un paciente infectado (Pizzi 1957) y tripomastigotes sanguíneos de la cepa colombiana Munantá, aislada originalmente de un vector Rhodnius prolixus en la región de Boyacá, Colombia, en 1995. Estas cepas de T. cruzi son mantenidas en nuestro laboratorio por pasajes sucesivos en ratones Balb/c.

Ratones. Se usaron ratones hembras, de la cepa ACA que son mantenidos por cruzamientos endogámicos en nuestro bioterio de cepas puras de ratones.

Modelos de infección. Grupos de 16 animales fueron usados en cada uno de los esquemas de trabajo: Un primer grupo de 2 meses de edad, fue inoculado con 2.000 parásitos/ratón de la cepa virulenta Tulahuen, por vía intraperitoneal (i.p.). Un segundo grupo, de 2 meses de edad, fue infectado i.p. con 2.000 tripomastigotes de la cepa no virulenta Munantá. Un tercer y último grupo de ratones de 1 mes de edad fue primero infectado con la cepa Munantá, y luego reinfectado, 5 semanas más tarde, con 2.000 parásitos de la cepa Tulahuen. La diferencia de edad de los dos primeros grupos con el tercero era necesaria para que en el momento de la reinfección con la cepa Tulahuen los ratones de este último grupo tuvieran también dos meses de edad.

Estudio de parasitemia. Cada dos días, a partir del segundo día postinfección, los niveles de parasitemia fueron analizados periódicamente hasta que los animales se hicieron completamente negativos. Para la determinación de la presencia y número de parásitos se tomaron muestras de sangre de la vena caudal de cada ratón en tubos de microhematocrito heparinizados, por capilaridad. El recuento de parásitos se realizó de acuerdo al método descrito por Arias y Ferro (1988).

Estudio histopatológico. Del grupo de ratones infectados sólo con parásitos de la cepa Tulahuen o de los infectados sólo con la cepa Munantá, se sacrificaron dos animales, a los días 5, 10 y 15 postinfección. En el caso de los animales infectados con Munantá y luego reinfectados con Tulahuen, se tomaron muestras de tejido a los días 5, 10, 15 y 30 postinfección. A cada uno de los animales sacrificados se le extrajo el corazón para estudio histopatológico y el tejido fue primero fijado en Bouin, formalina, y luego incluido en parafina según métodos convencionales. Finalmente, cortes de tejido de $8 \mu \mathrm{m}$ de espesor se tiñeron con hematoxilina-eosina. La severidad de la infiltración inflamatoria y el daño tisular fueron expresados arbitrariamente según una escala de tres puntos y considerando la infiltración leucocitaria, hiperemia, edema, hemorragia, pérdida de estriación y necrosis observada en el tejido: $(+)$ lesiones inflamatorias mínimas; $(++)$ lesiones moderadas; $(+++)$ lesiones severas con gran cantidad de infiltrado mononuclear. El nú- mero de células parasitadas (pseudoquistes) fue calificado de la siguiente manera: (+) presencia de 1-5 células parasitadas; (++) 6-10 pseudoquistes; (+++) más de 10 células parasitadas.

\section{RESULTADOS Y DISCUSION}

Los ratones ACA infectados con 2.000 parásitos de las cepas Tulahuen y Munantá de T. cruzi mostraron un período de prepatencia similar de 5 días, pero la mortalidad y el desarrollo de la parasitemia fueron diferentes. Animales infectados sólo con Tulahuen se mostraron como altamente susceptibles a la infección, con un $100 \%$ de mortalidad acumulada al día 20 postinfección, con una parasitemia positiva hasta el día 17 postinfección. Sin embargo, los animales inoculados con Munantá fueron resistentes a la infección pero con evidencia de parásitos circulantes hasta el día 28 postinfección. Al comparar los niveles máximos de parasitemia alcanzados, se pudo observar que los ratones infectados con la cepa Munantá desarrollaron niveles máximos mayores $\left(4,1 \times 10^{6}\right.$ parásitos/ml) que los alcanzados por los animales infectados con Tulahuen $\left(1,5 \times 10^{6}\right.$ parásitos $\left./ \mathrm{ml}\right)$ (figura 1).

Estos resultados muestran que la cepa Tulahuen de T. cruzi es altamente virulenta para ratones de la cepa ACA, produciendo la muerte del $100 \%$ de los animales dentro de las tres semanas postinfección, pero induciendo un nivel máximo de parasitemia bastante menor que el producido por la cepa colombiana Munantá, sobre la cual no existían antecedentes en modelos biológicos y que se comporta como no virulenta puesto que sobrevive el $100 \%$ de los ratones infectados. Estos resultados estarían de acuerdo con la idea que no siempre existe una correlación entre los niveles de parasitemia y la resistencia o susceptibilidad a la infección con T. cruzi (Minoprio y col 1989, Hoft y col 1993, Zúñiga y col 2002).

En el cuadro 1 se muestran los resultados del análisis histopatológico de corazón de los diferentes grupos experimentales. Al día 5 postinfección, tanto los ratones infectados con la cepa Munantá como aquellos infectados con Tulahuen sólo muestran lesiones moderadas, principalmente hiperemia y edema en pericardio. En el día 10 postinfección se mantienen estas características histopatológicas en ambos grupos, pero se observa una infiltración por células mononucleares y hemorragia más severa en el tejido de ratones infectados con Tulahuen. Al día 15 postinfección se pudo determinar la presencia de grados variables de infiltración mononuclear e hiperemia, pero sólo el pericardio de los animales infectados con Tulahuen mostró zonas hemorrágicas. No se encontraron pseudoquistes en el pericardio de los ratones infectados con la cepa Munantá y tampoco en aquellos infectados con Tulahuen. En miocardio, al día 5 postinfección fueron evidentes la hiperemia, edema y pérdida de la estriación de las fibras musculares sólo en el 


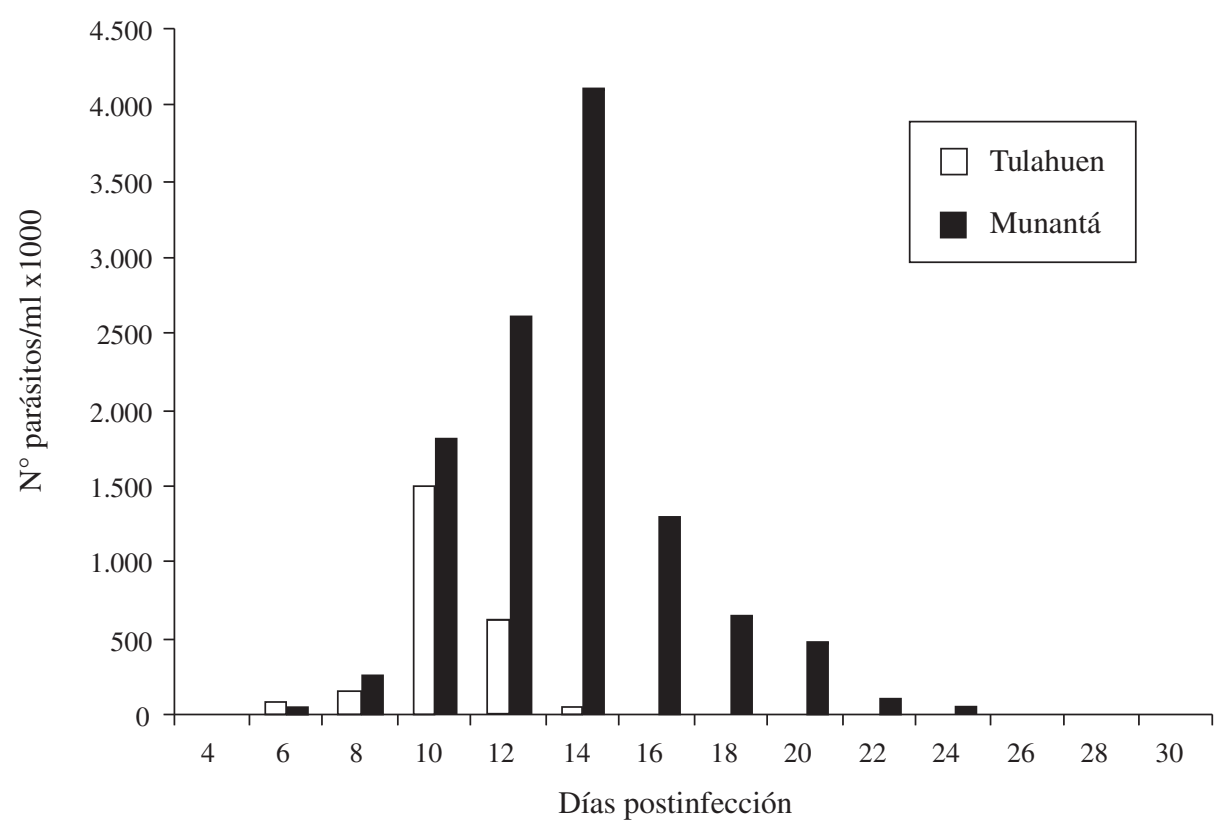

Figura 1. Parasitemia en ratones ACA infectados con 2.000 tripomastigotes de las cepas Tulahuen y Munantá de T. cruzi.

Parasitaemia in ACA mice infected with 2,000 trypomastigotes from Munantá (M) and Tulahuen (T) Trypanosoma cruzi strains.

Cuadro 1. Estudio histopatológico de corazón de ratones ACA infectados con 2.000 tripomastigotes de las cepas Munantá (M) y Tulahuen (T) de Trypanosoma cruzi.

Histopathological study in heart of ACA mice infected with 2,000 trypomastigotes from the Munantá (M) and Tulahuen (T) Trypanosoma cruzi strains.

\begin{tabular}{|c|c|c|c|c|c|c|c|c|c|c|}
\hline \multirow[b]{4}{*}{ Pericardio } & \multicolumn{6}{|c|}{ Ratones infectados } & \multicolumn{4}{|c|}{ Ratones reinfectados } \\
\hline & \multicolumn{10}{|c|}{ Días postinfección } \\
\hline & \multicolumn{2}{|c|}{5} & \multicolumn{2}{|c|}{10} & \multicolumn{2}{|c|}{15} & \multirow{2}{*}{$\frac{5}{\mathrm{~T}}$} & \multirow{2}{*}{$\frac{10}{\mathrm{~T}}$} & \multirow{2}{*}{$\frac{15}{\mathrm{~T}}$} & \multirow{2}{*}{$\frac{30}{\mathrm{~T}}$} \\
\hline & $\mathrm{T}$ & M & $\mathrm{T}$ & M & $\mathrm{T}$ & M & & & & \\
\hline Infiltración leucocitaria & + & + & +++ & + & ++ & + & + & ++ & + & - \\
\hline Hiperemia & ++ & ++ & +++ & +++ & +++ & ++ & +++ & ++ & + & + \\
\hline Edema & ++ & + & ++ & ++ & + & + & + & - & - & - \\
\hline Hemorragia & - & - & +++ & + & +++ & - & - & - & - & - \\
\hline Pseudoquistes & - & - & - & - & - & - & - & - & - & - \\
\hline \multicolumn{11}{|l|}{ Miocardio } \\
\hline Infiltración leucocitaria & + & - & ++ & + & +++ & + & - & + & + & - \\
\hline Hiperemia & ++ & + & ++ & +++ & ++ & + & - & + & + & - \\
\hline Edema & ++ & + & ++ & + & + & - & + & + & - & - \\
\hline Hemorragia & - & - & +++ & + & + & - & - & - & - & - \\
\hline Pérdida de estriación & ++ & - & ++ & + & +++ & + & + & - & - & - \\
\hline Necrosis & - & - & - & - & ++ & - & - & - & - & - \\
\hline Pseudoquistes & - & - & - & - & +++ & + & - & - & - & - \\
\hline
\end{tabular}


caso de la infección con Tulahuen. Al día 10 postinfección se mantienen las condiciones anteriores y se agrega la aparición de zonas hemorrágicas. En el día 15 de estudio, los tejidos de los animales infectados con Tulahuen mostraron gran infiltrado mononuclear, pérdida de estriación, hiperemia y focos necróticos (figura 2). Por el contrario, los tejidos de los animales infectados con Munantá presentaron sólo alteraciones leves (figura 3). Por otro lado, sólo en este día 15 postinfección se observaron pseudoquistes en ambas situaciones, pero con la diferencia de que mientras en los tejidos de los animales infectados con Tulahuen se observaron 58 pseudoquistes y una intensa infiltración leucocitaria, en las muestras de los ratones infectados con Munantá sólo se observó un pseudoquiste y ausencia de un fenómeno inflamatorio evidente. En resumen, los ratones infectados con Tulahuen mostraron alteraciones cardíacas severas con miocarditis, abundante infiltración mononuclear, alto número de pseudoquistes en corazón y 100\% de mortalidad. Por el contrario, la infección con la cepa Munantá determinó un curso más suave del proceso inflamatorio, menor número de pseudoquistes y $100 \%$ de sobrevivencia.

El análisis histopatológico permitió confirmar que, como ya se había sugerido, el parasitismo intracelular y el infiltrado inflamatorio varían de acuerdo a la cepa de parásito utilizada en la infección experimental (Rowland y col 1992). Los resultados muestran una correlación entre el número de parásitos circulantes, parasitismo intracelular y daño tisular sólo en el caso de la infección con la cepa Tulahuen. La infección con la cepa Munantá indujo altos niveles de parasitemia, pero con una escasa presencia de parásitos en el miocardio. Lo anterior podría explicarse

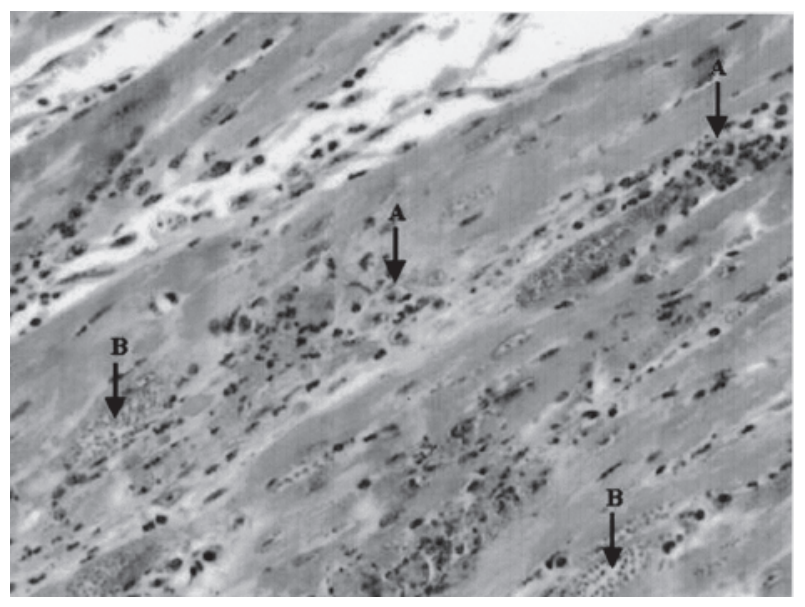

Figura 2. Daño tisular a los 15 días postinfección en miocardio de ratones infectados con la cepa Tulahuen. Se observa infiltrado mononuclear (A) y pseudoquistes (B), además de hiperemia y edema $(20 \mathrm{x})$.

Tissue damage at 15 days postinfection in myocardial tissue sections of mice infected with the Tulahuen strain. Mononuclear infiltrate and pseudocysts besides hyperaemia and oedema were observed (20x).

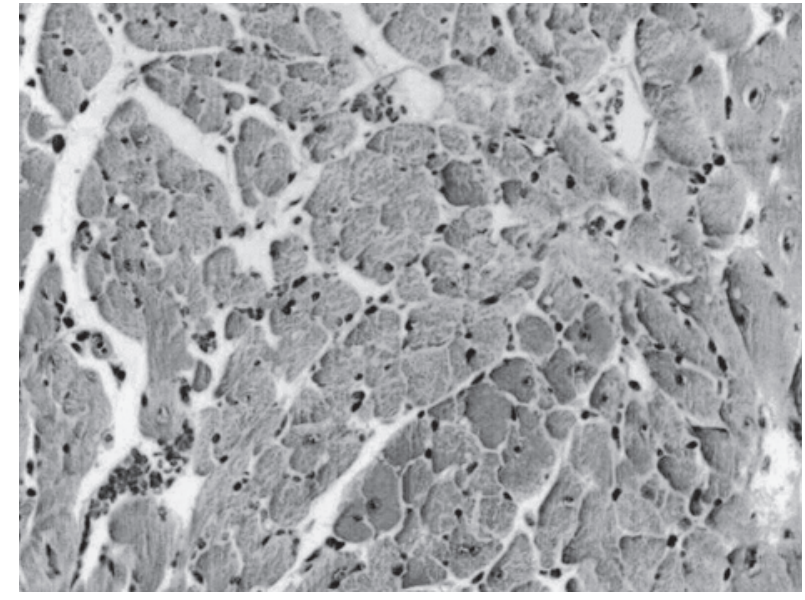

Figura 3. Daño tisular a los 15 días postinfección en miocardio de ratones infectados con la cepa Munantá. Se observa ausencia de lesiones inflamatorias y de pseudoquistes $(20 \mathrm{x})$.

Tissue damage at 15 days postinfection in myocardial tissue sections of mice infected with the Munantá strain. Absence of inflammatory lesions and pseudocysts were observed (20x).

porque esta cepa colombiana tendría un tropismo hacia tejidos diferentes de miocardio (Melo y Brener 1978), ya que hasta ahora no se ha demostrado que $T$. cruzi pueda replicarse en el torrente sanguíneo como ocurre con los tripanosomas africanos.

En los ratones previamente infectados con la cepa Munantá la reinfección con la cepa Tulahuen, 5 días después de desaparecida la parasitemia al día 30 postinfección, mostró una disminución en la severidad de la infección con $100 \%$ de sobrevivencia, menor daño tisular (cuadro 1) (figura 4) y menores niveles de

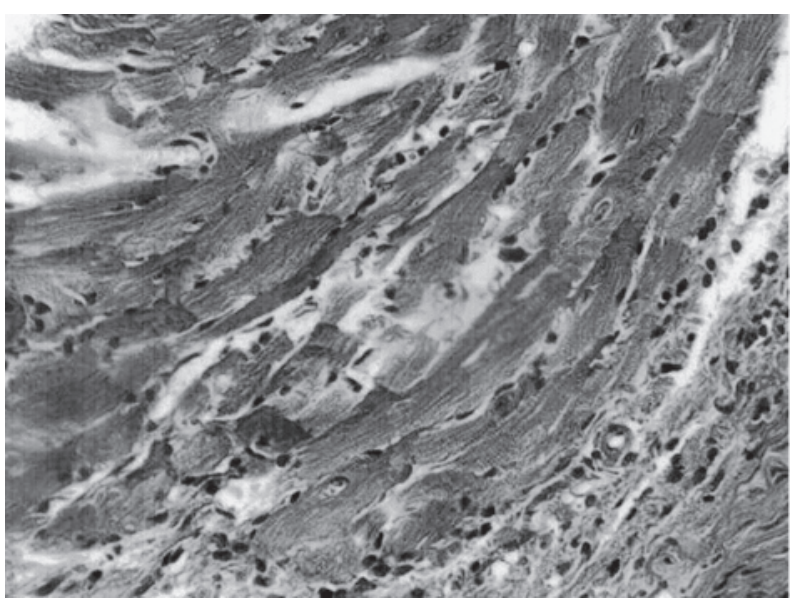

Figura 4. Daño tisular a los 15 días postreinfección con la cepa Tulahuen en miocardio de ratones previamente infectados con la cepa Munantá. Se observan leves lesiones inflamatorias, ausencia de pseudoquistes y escaso infiltrado mononuclear $(20 \mathrm{x})$.

Tissue damage at 15 days post-reinfection in myocardial tissue sections of mice infected with Tulahuen strain previously infected with the Munantá strain. Moderate inflammatory lesions, absence of pseudocysts and mild cellular infiltrate were observed (20x). 
parasitemia $\left(6,5 \times 10^{4}\right.$ parásitos $\left./ \mathrm{ml}\right)$ que los ratones infectados sólo con la cepa Tulahuen (1,5 x $10^{6}$ parásitos/ $\mathrm{ml}$ ), sugiriendo que la infección con una cepa no virulenta de Trypanosoma cruzi induce una respuesta que altera el curso de la infección al influir o modificar los niveles de parasitemia, el daño tisular y la respuesta inmune contra cepas virulentas del parásito.

El estudio de la enfermedad de Chagas en modelos animales ha permitido establecer la existencia de distintos mecanismos de patogénesis, incluyendo inflamación inducida por antígenos parasitarios, daño tisular en el miocardio y reparación y autoinmunidad (Leon y Engman 2001). En el modelo murino se observan diferencias en el curso de la infección dependiendo de la cepa de ratón y de la virulencia del aislado o cepa de Trypanosoma cruzi, y un único clon parasitario puede inducir un curso distinto de la enfermedad en ratones susceptibles o resistentes a la infección (Leon y Engman 2001). Por otro lado, estudios con Trypanosoma rangeli, una especie distinta de tripanosoma y no patógena para mamíferos (Mansfield 1977), han mostrado un efecto protector de T. rangeli en infecciones mixtas T. cruzi/T. rangeli (Basso y col 1989, Zúñiga y col 1997) y se ha sugerido que ello obedece a similitudes antigénicas entre estas dos especies de tripanosoma (Guhl y col 1985, Saldaña y Sousa, 1996).

En nuestro caso se debería esperar una mayor similitud antigénica entre las cepas Tulahuen y Munantá, puesto que ambas constituyen variantes de T. cruzi. Por lo tanto, es probable que una respuesta inmune contra la cepa Munantá desarrolle inmunidad cruzada para un mejor control de la infección con parásitos de la cepa Tulahuen en los ratones experimentalmente inoculados con ambos parásitos.

Es sabido que la enfermedad de Chagas presenta un amplio rango de manifestaciones clínicas, que puede ir desde formas asintomáticas hasta los característicos megasíndromes (Botero y Restrepo 1992). En países como Colombia y Venezuela, T. cruzi coexiste con $T$. rangeli en vectores y hospederos vertebrados y es precisamente en estos países donde la sintomatología de la enfermedad es menos definida y con escasa presencia de megasíndromes (Aragort de Rossel y col 2000), los cuales son más habituales en países como Chile y Argentina, donde no existe $T$. rangeli. La posible modificación de la infección chagásica en infecciones mixtas, con cepas virulentas y no virulentas, parece un aspecto relevante en el avance del conocimiento sobre la patogenia y control de una enfermedad, para la cual no existe, hasta ahora, una vacuna disponible ni tratamiento altamente efectivo. La identificación de los antígenos de cepas no virulentas, involucrados en la inducción de una respuesta inmune protectora contra la infección con cepas virulentas del parásito y por lo tanto capaces de interferir con el curso de la infección y el daño celular autoinmune asociado a la enfermedad, parece crucial para el desarrollo de una eventual vacuna contra Trypanosoma cruzi.

\section{RESUMEN}

En el presente estudio se realizó una comparación en el desarrollo de la infección con 2.000 tripomastigotes, en ratones ACA, usando las cepas Tulahuen y Munantá de Trypanosoma cruzi, aisladas originalmente en Chile y Colombia, respectivamente. La cepa ACA se mostró como altamente susceptible a la infección con la cepa Tulahuen, muriendo el $100 \%$ de los animales dentro de las 3 semanas postinfección. Sin embargo, todos los ratones infectados con la cepa Munantá sobrevivieron a pesar de alcanzar mayores niveles de parasitemia. El estudio histopatológico de corazón mostró diferentes grados de parasitismo intracelular e infiltrado inflamatorio en relación con la cepa de parásito utilizada. En la infección con la cepa Munantá se pudo observar escasa presencia de pseudoquistes, en este tejido, con una discreta inflamación. Por el contrario, los tejidos de los animales infectados con la cepa Tulahuen mostraron un alto número de parásitos intracelulares y un severo proceso inflamatorio. Se investigó el posible efecto protector de una primoinfección con la cepa no virulenta Munantá frente a una posterior infección con la cepa virulenta Tulahuen. Los animales reinfectados con esta última cepa mostraron menores niveles de parasitemia, $100 \%$ de sobrevivencia y menor daño tisular.

\section{REFERENCIAS}

Aragort de Rossel R, A Rodríguez, R De Jesus, M Calcagno, Z Maizo, S Diaz. 2000. Tripomastigotes de sangre y de cultivo celular de Trypanosoma cruzi. Parasitol Día. 3-4, 79-97.

Arias A, E Ferro. 1988. Quantification of Trypanosoma cruzi parasitemia by direct micromethod. Trans Roy Soc Trop Hyg 82, 248.

Basso B, E Moretti, S Fontenia, E Vottero-Cima. 1989. Trypanosoma cruzi and Trypanosoma rangeli: overlapping of antigenic spectrum. Rev Latinoam Microbiol 2, 141-146.

Botero D, P Restrepo. 1992. Parasitosis humanas. $1^{\text {a }}$ ed. Corporación para Investigaciones Biológicas. Medellín, Colombia.

Burleigh B, N Andrews. 1995. The mechanisms of the Trypanosoma cruzi invasion of mammalian cells. Ann Rev Microbiol 49, 175-200.

Dias J, A Silveira, CJ Schofield. 2002. The impact of Chagas disease control in Latin America. Mem Inst Oswaldo Cruz 97, 603-612.

Guhl F, L Hudson, C Marinkelle, S Morgan, C Jaramillo. 1985. Antibody response to experimental. Trypanosoma rangeli infections and its implications for immunodiagnosis of South American trypanosomiasis. Acta Tropica 42, 311-318.

Hoft D, R Lynch, L Kirchoff. 1993. Kinetics analysis of antigenspecific immune response in resistant and susceptible mice during infection with Trypanosoma cruzi. J Immunol 151, 7038-7047.

Leon JS, DM Engman. 2001. Autoimmunity in Chagas heart disease. Int J Parasitol. 31, 555-561.

Mansfield J. 1977. Non pathogenic trypanosomes of mammals. In: Parasitic Protozoa. Academic Press, New York, U.S.A

Melo R, Z Brener. 1978. Tissue tropism of different Trypanosoma cruzi strains. J Parasitol 64, 475-482.

Minoprio P, S Itohara, C Heusser, S Tonegawa, A Coutinho. 1989. Immunobiology of murine $T$. cruzi Infection: The predominance of parasite-nonspecific response and the activation of TCRI cells. Immunol Rev 12, 183-207.

Morris S, H Tanowitz, M Wittner, J Bilezikian. 1990. Pathophysiological insights into the cardiomyopathy of Chagas disease. Circulation 82, 1900-1909.

Pizzi T. 1957. Inmunología de la Enfermedad de Chagas. Universidad de Chile. Santiago, Chile.

Postan M, J Dvorak, J Mc Daniel. 1983. Studies of Trypanosoma cruzi clones in inbred mice. Am J Trop Med Hyg 32, 497-506.

Rowland E, M Lozykowski, T Mc Cormick. 1992. Differential cardiac histopathology in inbred mouse strains chronically infected with Trypanosoma cruzi. J Parasitol 78, 6-10. 
Saldaña A, Sousa O. 1996. Trypanosoma rangeli and Trypanosoma cruzi: cross-reaction among their immunogenic components. Mem Inst Oswaldo Cruz 1, 81-82.

Stevens J, H Noyes, JC Schofield, W Gibson. 2001. The Molecular evolution of Trypanosomatidae. Adv. Parasitol. 48, 1-53.

Urzúa C, MA Morales, U Vergara, MT Paláu, C Zúñiga. 2004. Sexo del hospedero y dosis infectante de parásitos como factores en el desarrollo de la infección con Trypanosoma cruzi en un modelo murino. Parasitol Latinoam 3-4, 104-109.
Zúñiga C, MT Paláu, P Penin, C Gamallo, JA De Diego. 1997. Protective effect of Trypanosoma rangeli against infection with a highly virulent strain of Trypanosoma cruzi. Trop Med Int Health 5, 482-487.

Zúñiga C, A Parra, H Vela, T Courcelles, R Vargas, U Vergara. 1998. Estudio histopatológico en ratones infectados en forma experimental con Trypanosoma cruzi. Parasitol Día 22, 23-28.

Zúñiga C, R Vargas, U.Vergara. 2002. Evolución de la infección con Trypanosoma cruzi en cepas susceptibles y resistentes de ratones. Arch Med Vet 2, 183-188. 